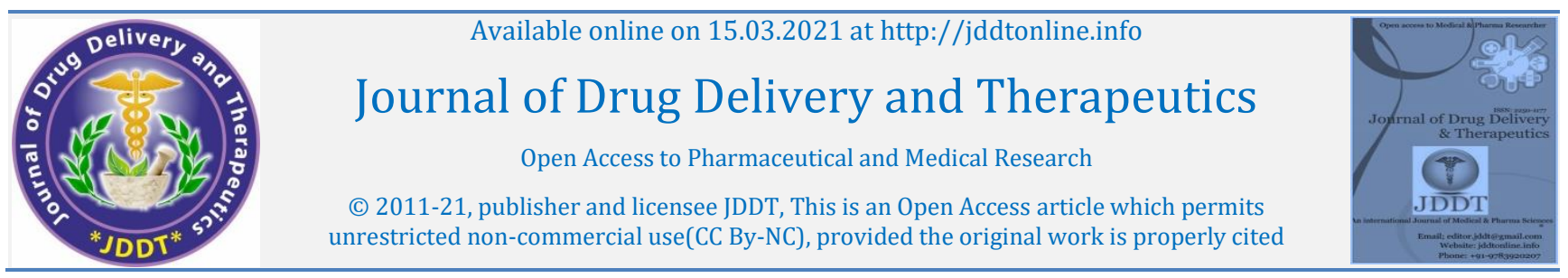

Open Access Full Text Article

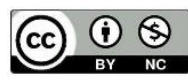

Research Article

\title{
Assessment of Predictors of Hyperkalemia in Cardiovascular Diseased Patients
}

\author{
Manasa R*, Aksa Johnson, Presly Thomas Augustine, Anjana Tom \\ Department of Pharmacy practice, Bapujji Pharmacy College, Shamnur Road, S.S Layout, Davangere-577004, Karnataka, India
}

\section{Article Info:}

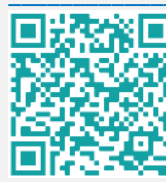

\section{Article History:}

Received 11 Jan 2021;

Review Completed 19 Feb 2021

Accepted 26 Feb 2021.

Available online 15 March 2021

\section{Cite this article as:}

R M, Johnson A, Augustine PT, Tom A, Assessment of Predictors of Hyperkalemia in Cardiovascular Diseased Patients, Journal of Drug Delivery and Therapeutics. 2021; 11(2):51-54

DOI: http://dx.doi.org/10.22270/jddt.v11i2.4587

*Address for Correspondence:

Manasa R, Department of Pharmacy practice, Bapujji Pharmacy College, Shamnur Road, S.S Layout, Davangere-577004, Karnataka, India

\section{Abstract}

Purpose: The objective is to determine the predictors of hyperkalaemia, severity of hyperkalaemia and to access the prevalence of cardiovascular diseases

Methodology: A cross sectional observational study was conducted on the predictors of hyperkalaemia in CVD patients, in a tertiary care teaching hospital for a period of 6 months.

Results: Among 105 patients, there were 83 males and 22 females, the age group from 41-60 year shows more CVDs and 61-80 years aged patients are more prone to hyperkalaemia and shows more prevalent when compared to other age groups. The major risk factors of hyperkalaemia were Drugs (30.23\%), Disease (58.13\%), and Drug interactions (11.62\%).Among the cardiovascular diseases, Myocardial Infarction (38\%) were the most common type of CVD, while in hyperkalaemia Ischemic Heart Disease (48.83\%) were more prevalent. The severity of hyperkalaemia is measured as Mild (60.46\%), Moderate (37.20\%), and Severe $(2.32 \%)$.

Conclusion: The present study was carried out in order to assess the predictors, prevalence and severity of hyperkalaemia in cardiovascular disease patients. Our study concluded that patients with cardiovascular disease can have an increased risk of hyperkalaemia.

Keywords: CVD, Hyperkalaemia, Cross sectional observational study, Predictors, Prevalence, Severity.

\section{INTRODUCTION}

Hyperkalaemia is the medical term that describes a potassium level in your blood that is higher than normal (3.5-5.5mEq/L) ${ }^{1}$.Globally hyperkalaemia has been reported in less than $5 \%$ of the general population, an estimated 3.7 million adults had hyperkalaemia in 2010 and this prevalence rate has increased since 2014 to $11.5 \%$ hence it increases the chance of risk in $\mathrm{CVD}^{1}$

Mild cases of hyperkalaemia may not produce symptoms and may be easy to treat, severe cases of hyperkalaemia that are left untreated can lead to fatal cardiac arrhythmias, which are abnormal heart rhythms ${ }^{3}$. The cardiac toxicity of hyperkalaemia is a major cause of morbidity and mortality 4 , Hence we are finding the risk factors of hyperkalaemia that contribute towards cardiovascular disease; such as Disease, Drug, and Drug interaction ${ }^{1}$. Hyperkalaemia is the most common condition and it has been discovered on routine laboratory tests. Patients with heart failure (HF), diabetes mellitus (DM), and drugs like ACE inhibitors, reninangiotensin-aldosterone system inhibitors (RAASi), spironolactone, and these agents alone or in combination cause elevation in potassium, and they are at higher risk for hyperkalaemia ${ }^{2}$

Potassium is the major intracellular cation in the body. In that majority of potassium is sequestered within the cell,
Potassium and sodium concentrations play a crucial role in the electric signal functioning of the heart's middle layer known as the myocardium. An increased level of potassium can interfere with the electrical signals in the muscle layer and that decreases the intracellular/extracellular potassium ratio. Hence the resting membrane potential becomes less negative and closer to the threshold excitation potential and leads to different types of heart arrhythmias ${ }^{3}$. So estimating the additional risk factors of hyperkalaemia would be helpful to clinicians for risk assessment and to the addition of highrisk patients 4 .

Based on severity, hyperkalemia is classified as mild (5.5$6.0 \mathrm{mEql})$, moderate $(6.1-6.9 \mathrm{mEq} / \mathrm{l})$ severe $(>7.0 \mathrm{mEq} / \mathrm{l})^{16}$. The patients, by virtue of their disease, comorbidities, and medical therapy, are at risk for hyperkalemia. Hyperkalemia can be classified into two types:

1. Inherent hyperkalemia: includes hormonal disorders (e.g., Addison's disease, Hyporeninemic Hyperaldosteronism), diabetes mellitus, and diseases with cell membrane instability that can cause intracellular and extracellular shifts;

2. Treatment-related hyperkalemia: medications (e.g., RAASi, mineralocorticoid receptor antagonists, NSAIDs, diuretic agents, heparin) ${ }^{1}$. 


\section{MATERIALS AND METHODS:}

The study was conducted in inpatient wards of the department of cardiology 500 bedded teaching hospital. This was an cross sectional observational study that was conducted for a 6-month period. The subjects who were of age 18 years and above, all inpatients having cardiovascular disease and subjects who were willing to participate in the study and signed written informed consent were included. Lactating and pregnant women up to 12 weeks after Partum and subjects with psychiatric disorders and those who were unable to answer the questions and all patients with renal impairment were excluded.

Data was collected from the selected subjects. Subjects were also counselled about their disease during their hospitalization. Collected data were entered in Microsoft Excel to evaluate the objectives of the study.

\section{Study Tools:}

Patient's social history, location, and demographic details; clinical data including duration of hospital stay, radiographic details, laboratory profile, diagnosis, symptoms, etc. therapeutic data including the name, route, dose, and frequency of the drug; the duration of therapy and other relevant details were recorded in a suitably-designed individual case record form by reviewing their prescriptions, medical records, and caretakers.

\section{Ethical Clearance}

The study was approved by BAPUJI PHARMACY COLLEGE institutional ethical committee on human subjects research with approval number IEC No; BPC/IEC No.58/2019-2020

Subjects meeting the inclusion and exclusion criteria who hospitalised in cardiology department were explained about the purpose of the study and informed consent was obtained. Data was collected from the selected subjects with the help of a self-designed data collection form. It was then used to obtain information about predictors of hyperkalemia in cardiovascular disease patients. Subjects were also counselled about their disease during their hospitalisation. Collected data were entered in Microsoft Excel to evaluate the objectives of the study.

\section{RESULTS:}

The study included a total of 105 patients were enrolled in the study, out of which 83 (79\%) were Males and 22 (20.9\%) were Females (fig.1).

The resulted of Age-wise categorization revealed that out of 105 subjects 52 ( $49.5 \%$ ) patients belong to the class of 41 60 years, followed by $35(33.3 \%)$ patients are in the age group of $61-80$ years, $11(10.6 \%)$ patients were between 18 40 years, only 7 ( $6.61 \%$ ) were aged more than 80 years, as mentioned in Table 1.
Gender Distribution of Hyperkalemic patients

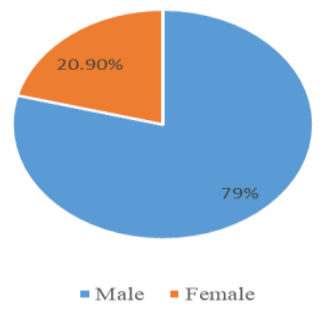

Figure 1: Gender distribution of hyperkalemia patients

Table 1: Distribution of Hyperkalemic Patients based on Age Group

\begin{tabular}{|l|l|l|l|}
\hline S.N. & Age category & $\begin{array}{l}\text { No. of } \\
\text { patients }\end{array}$ & $\begin{array}{l}\text { Percentage } \\
\text { (\%) }\end{array}$ \\
\hline 1 & $18-40$ & 11 & 10.6 \\
\hline 2 & $41-60$ & 52 & 49.5 \\
\hline 3 & $61-80$ & 35 & 33.3 \\
\hline 4 & $>80$ & 7 & 6.6 \\
\hline
\end{tabular}

Figure 2. Illustrates that predictors of hyperkalemia were a disease, drug, and drug interaction. There were $61.9 \%$ patients with hyperkalemia associated with known Disease ( IHD,HTN, MI, DM,RA) , 32 patients ( $30.47 \%$ ) with Drugrelated hyperkalemia (ACE,ARB, Spironolactone) , 08 patients $(7.62 \%)$ with Drug interaction (Spironolactone+ACE, ACE+ARB) associated with hyperkalemia.

Distribution of Hyperkalemic patients based on Risk Factors

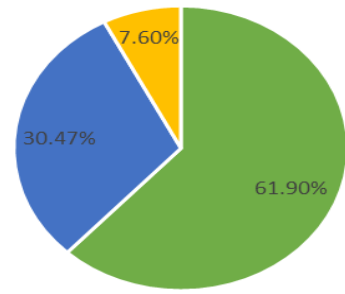

- DISEASE - DRUGS = DRUG INTERACTION

Figure 2: Distribution of Hyperkalaemia patients based on Risk Factors

As depicted in Table 2, Among the 105 hyperkalemic patients, the prominent disease was IHD $45(42.83 \%)$ patients, followed by MI 36(34.28\%), Unstable angina $9.30 \%$ and other conditions with $4.7 \%$ of total subjects diagonised with hyperkalemia.

Table 2: Distribution of Cardiovascular diseases among Hyperkalemic patients

\begin{tabular}{|l|l|l|l|}
\hline Sl.no & Cardiovascular Disease & No. of Patients(n=105) & Percentage (\%) \\
\hline 1 & IHD & 45 & 42.8 \\
\hline 2 & MI & 36 & 34.28 \\
\hline 3 & Unstable Angina & 08 & 7.6 \\
\hline 4 & CAD & 04 & 3.80 \\
\hline 5 & CHD & 03 & 2.8 \\
\hline 6 & Arrhythmia & 04 & 3.8 \\
\hline 7 & Others & 05 & 4.7 \\
\hline
\end{tabular}


The results of the study on the basis of severity of hyperkalemia illustrated that most of the patients belong to Mild category 26 $(60.46 \%)$, Moderate $16(37.20 \%)$ and Severe $1(2.32 \%)$ in nature as shown figure 3.

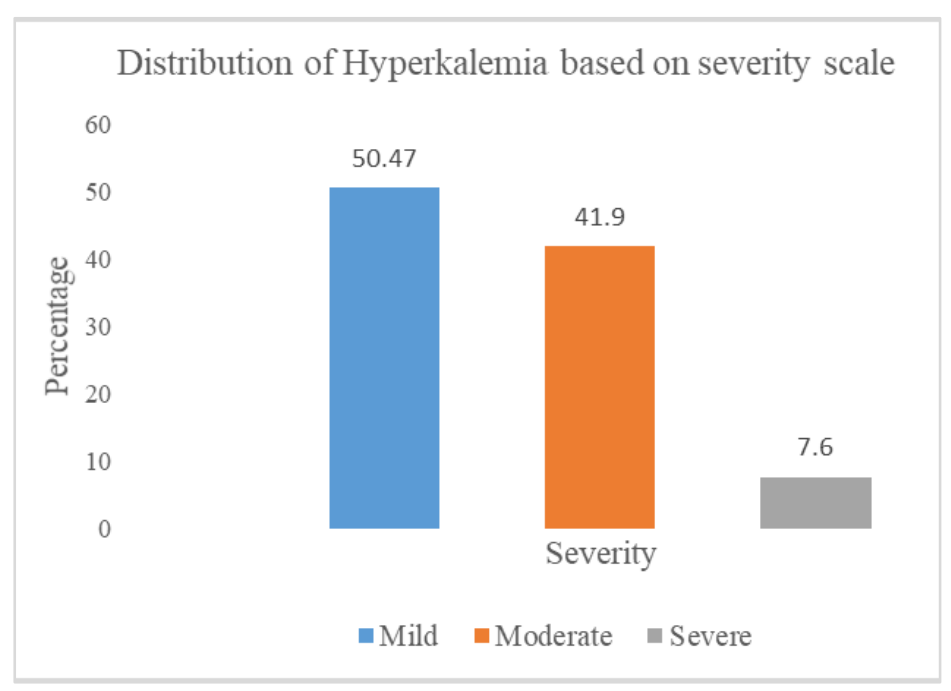

Figure 3: Distribution of Hyperkalaemia based on severity scale

\section{DISCUSSION}

Cardiovascular diseases are a group of disorders of heart and blood vessels. CVDs are one of the leading causes of death in India due to change in food habits, sedentary lifestyle etc. There were certain risk factors for CVD such as high BP, Smoking, DM, electrolyte changes etc. Hyperkalemia is one of the risk factors for CVD. The objective of our study was to assess risk factors of hyperkalemia among people with cardiovascular disease.

This study was conducted for a period of 6 months, a cohort of 105 patients with cardiovascular disease were assessed for the risk factors of hyperkalemia. Based on the patient demographic details, past medical and medication history and laboratory parameters (electrolytes) of the patients were assessed to meet the objectives of the study.

A total of 105 patients were enrolled in the study, out of which 83 (79\%) were Males and 22 (20.9\%) were Females. This study also reveals males predominance over female and was similar to the study conducted by Aishwarya A.T, et-al ${ }^{1}$

The present study demonstrated that, the most prevalent age group with cardiovascular disease belongs to 41-60 years of age $(45 \%)$ which is contrary to the study conducted by Aishwarya A.T, et-al ${ }^{1}$ which demonstrated the age group of $61-70$, followed by $33.3 \%$ of patients are in the age group of 61-80 years, $10.6 \%$ patients were between $18-40$ years, and about $6.6 \%$ patients belonging to the age group of more than 80 years.

As depicted in Table 2, The most commonly seen cardiovascular disease among subjects were identified as Myocardial infarction (34.28\%), Ischemic heart disease $(42.8 \%)$, Unstable angina $(7.6 \%)$, Congestive heart failure $(2.8 \%)$, Coronary artery disease $(3.80 \%)$, Arrhythmia (3.80\%) and patients with Congestive heart disease $(2.9 \%)$ which is similar to the study conducted by Alexander Michel et $\mathbf{a l}^{5}$, which showed that $96.7 \%$ had IHD followed by other cardiac diseases. Whereas in a study conducted by Michael $\boldsymbol{H}$ Alderman et $\mathbf{a l}^{6}$ showed that (11.1\%) of coronary artery disease as the most common disease which is contradictory to the present study results.

The risk factors of hyperkalaemia among CVD patients were categorized as disease, drugs, and drug interaction induced, among the study population the predictors of hyperkalaemia was known to be is due to Disease (61.9\%), 30.47\% were Drug related, and $7.6 \%$ was due to Drug interaction. This study shows that the prominent risk factors include Hypertension, IHD, diabetes, IWMI, few drugs include ACE inhibitors, ARBs, Spironolactone and drug interactions includes ACE inhibitors with ARBs and ACE inhibitors with spironolactone. These drug-drug interactions were procured from Micromedex. The results of the study are similar to the study done by Alexander Michel et al $^{5}$ in their study results shows the significant risk factors of hyperkalaemia were renal failure, type 2 diabetes mellitus, valvular heart disease and current use of potassium sparing diuretics, trimethoprim, NSAIDs and several drug combinations.

The study also categorised the hyperkalemic patients based on the severity of the disease as Mild (5.5- $6.0 \mathrm{mEq} / \mathrm{L})$, Moderate (6.1-6.9mEq/L), Severe (>7.0 mEq/L). Our study showed that most hyperkalemic patients belong to Mild category (50.47\%) followed by Moderate (41.9.\%), and only $7.6 \%$ of patients were found to be Severe . which is similar to the study results conducted by Aiswarya $A$. T et al ${ }^{1}$.

\section{CONCLUSION}

The occurrence of hyperkalaemia in cardiovascular disease patients was described based on their severity, age group, gender and different cardiovascular conditions. Our findings may better help to identify patients with heart failure most likely to benefit from the careful monitoring of serum potassium levels. Pharmacovigilance is needed during the start of treatment with certain medications.

\section{ACKNOWLEDGEMENT:}

I'm thankful to Dr. Merin Titus, Assistant Professor at Bapuji College of Pharmacy, Davangere, Karnataka, for her valuable time to audit my paper and for her thoughtful suggestions.

\section{Conflict of Interest: None.}

Source of Funding: NO Funding. 


\section{REFERENCES:}

1. Aiswarya A T, et al. A Study on the Assessment of Risk Factors and Evaluation of Prescribing Pattern in Patients with Hyperkalemia Having Cardiovascular Disorders", International journal of pharmacy and pharmaceutical research,vol 2018; 11(4)

2 Núñez Julio et al. "Long-Term Potassium Monitoring And Dynamics In Heart Failure And Risk Of Mortality". Circulation, 2018, 137(13):1320-1330. Ovid Technologies (Wolters Kluwer Health), doi:10.1161/circulationaha.117.030576.

3. High potassium (hyperkalemia) [Internet] 2018 Jan 11. Available form: https://www.mayoclinic.org/symptoms/hyperkalemia/basics/ definition/sym-20050776

4. Alldredge B, Koda-Kimble M, Young L. Applied therapeutics. Philadelphia, Pa.: Wolters Kluwer / Lippincott Williams \& Wilkins; 2013

5. Michel Alexander et al. "Risk Factors For Hyperkalaemia In A Cohort Of Patients With Newly Diagnosed Heart Failure: A Nested Case-Control Study In UK General Practice". European Journal of -9-Heart Failure, 2015; 17(2):205-213. Wiley, doi:10.1002/ejhf.226.

6. Alderman Michael H. et al. "Clinical Significance Of Incident Hypokalemia And Hyperkalemia In Treated Hypertensive Patients In The Antihypertensive And Lipid-Lowering Treatment To Prevent Heart Attack Trial". Hypertension, 2012; 59(5):926933. Ovid Technologies (Wolters Kluwer Health), doi:10.1161/hypertensionaha.111.180554.

7. Ahee P. "The Management Of Hyperkalaemia In The Emergency Department". Emergency Medicine Journal, 2000; 17(3):188191. BMJ, doi:10.1136/emj.17.3.188.

8. Martín-Pérez Mar et al. "Impact Of Hyperkalaemia Definition On Incidence Assessment: Implications For Epidemiological Research Based On A Large Cohort Study In Newly Diagnosed Heart Failure Patients In Primary Care". BMC Family Practice, 2016; 17(1). Springer Science And Business Media LLC, doi: 10.1186/s12875-016-0448-5.

9. Amir Omalhassan et al. "Incidence Of Risk Factors For Developing Hyperkalemia When Using ACE Inhibitors In Cardiovascular Diseases". Pharmacy World \& Science, 2009; 31(3):387393. Springer Science And Business Media LLC, doi:10.1007/s11096-009-9288-x.

10.Vardeny, Orly et al. "Incidence, Predictors, And Outcomes Related To Hypo- And Hyperkalemia In Patients With Severe Heart Failure Treated With A Mineralocorticoid Receptor Antagonist". Circulation: Heart Failure, 2014; 7(4):573-579. Ovid Technologies (Wolters Health), doi:10.1161/circheartfailure.114.001104.

11. de Denus, Simon et al. "Quantification of the Risk and Predictors of Hyperkalemia in Patients with Left Ventricular Dysfunction". American Heart Journal, 2006; 152(4):705712. Elsevier BV, doi:10.1016/j.ahj.2006.05.030.

12. Desai, Akshay S. et al. "Incidence And Predictors Of Hyperkalemia In Patients With Heart Failure". Journal of The American College
Of Cardiology, 2007; 50(20):959-1966. Elsevier BV, doi:10.1016/j.jacc.2007.07.067.

13. Acker, Christopher G. et al. "Hyperkalemia In Hospitalized Patients". Archives of Internal Medicine, 1998; 158(8):917. American Medical Association (AMA), doi:10.1001/archinte.158.8.917.

14. Jain, Nishank et al. "Predictors of Hyperkalemia And Death In Patients With Cardiac And Renal Disease". The American Journal of Cardiology, 2012; 109(10):1510-1513. Elsevier BV, doi:10.1016/j.amjcard.2012.01.367.

15. Khanna, Apurv, and William B. White. "The Management of Hyperkalemia In Patients With Cardiovascular Disease". The American Journal of Medicine, 2009; 122(3):215-221. Elsevier $B V$, doi:10.1016/j.amjmed.2008.10.028.

16. Muzzarelli, Stefano et al. "Frequency And Predictors of Hyperkalemia In Patients $\geq 60$ Years Of Age With Heart Failure Undergoing Intense Medical Therapy". The American Journal of Cardiology, 2012; 109(5):693-698. Elsevier BV, doi:10.1016/j.amjcard.2011.10.027.

17. Palmer, Biff F. "Managing Hyperkalemia Caused By Inhibitors Of The Renin-Angiotensin-Aldosterone System". New England Journal of Medicine, 2004; 351(6):585-592. Massachusetts Medical Society, doi:10.1056/nejmra035279.

18. Toto, Robert D. "Serum Potassium And Cardiovascular Outcomes: The Highs And The Lows". Clinical Journal of The American Society Of Nephrology, 2017; 12(2):221. American Society Of Nephrology (ASN), doi:10.2215/cjn.00030117.

18 Tromp, Jasper et al. "Serum Potassium Levels And Outcome In Acute Heart Failure (Data From The PROTECT And $\mathrm{COACH}$ Trials)". The American Journal Of Cardiology, 2017; 119(2):290296. Elsevier BV, doi:10.1016/j.amjcard.2016.09.038.

19 Svensson, Morten et al. "How Prevalent Is Hyperkalemia And Renal Dysfunction During Treatment With Spironolactone In Patients With Congestive Heart Failure?". Journal of Cardiac Failure, 2004; 10(4):297-303. Elsevier BV doi:10.1016/j.cardfail.2003.10.012.

20 Thomsen, Reimar Wernich et al. "Elevated Potassium Levels In Patients With Congestive Heart Failure: Occurrence, Risk Factors, And Clinical Outcomes". Journal of the American Heart Association, 2018; 7(11). Ovid Technologies (Wolters Kluwer Health), doi:10.1161/jaha.118.008912.

21 Ahuja, Tejinder S. et al. "Predictors of The Development Of Hyperkalemia In Patients Using Angiotensin-Converting Enzyme Inhibitors". American Journal Of Nephrology, 2000; 20(4):268272. S. Karger AG, doi:10.1159/000013599.

22 Ramadan, F. H. et al. "Clinical Factors Associated With Hyperkalemia In Patients With Congestive Heart Failure1". Journal of Clinical Pharmacy And Therapeutics, 2005; 30(3):233-239. Wiley, doi:10.1111/j.1365-2710.2005.00638.x.

23 "Cardiovascular Diseases (Cvds)". Who.Int, 2021, https://www.who.int/news-room/factsheets/detail/cardiovascular-diseases-(cvds). 